\title{
OPEN Fabrication and characterization of resistive double square loop arrays for ultra-wide bandwidth microwave absorption
}

\author{
Ji-Young Jeong ${ }^{1,2}$, Je-Ryung Lee ${ }^{3}$, Hyeonjin Park ${ }^{4}$, Joonkyo Jung ${ }^{4}$, Doo-Sun Choi ${ }^{2}$, \\ Eun-chae Jeon ${ }^{5}$, Jonghwa Shin ${ }^{4}$, Jun Sae Han ${ }^{1,2,6 \bowtie} \&$ Tae-Jin Je ${ }^{1,2,6 \bowtie ~}$
}

Microwave absorbers using conductive ink are generally fabricated by printing an array pattern on a substrate to generate electromagnetic fields. However, screen printing processes are difficult to vary the sheet resistance values for different regions of the pattern on the same layer, because the printing process deposits materials at the same height over the entire surface of substrate. In this study, a promising manufacturing process was suggested for engraved resistive double square loop arrays with ultra-wide bandwidth microwave. The developed manufacturing process consists of a micro-end-milling, inking, and planing processes. A 144-number of double square loop array was precisely machined on a polymethyl methacrylate workpiece with the micro-end-milling process. After engraving array structures, the machined surface was completely covered with the developed conductive carbon ink with a sheet resistance of $15 \Omega / \mathrm{sq}$. It was cured at room temperature. Excluding the ink that filled the machined double square loop array, overflowed ink was removed with the planing process to achieve full filled and isolated resistive array patterns. The fabricated microwave absorber showed a small radar cross-section with reflectance less than $-10 \mathrm{~dB}$ in the frequency band range of $8.0-14.6 \mathrm{GHz}$.

Recently, with increasing importance of electronic warfare in the military field, microwave absorbers with perfect absorption against incident electromagnetic waves are vigorously studied. The conventional method for absorbing a microwave is using a paint that contains tiny spheres made of carbonyl iron or ferrite. These particles convert microwave energy to heat through coupled electromagnetic oscillations ${ }^{1-4}$. However, the required minimum thickness of paint for a high absorptance and low reflectance is considerably high. Because ferrite materials have a high specific gravity, thick ferrite paints can increase the weight of the applied body significantly. For this reason, light and thin absorbers that can strongly absorb microwave without using heavy ferrite materials are in demand. Such needs have resulted in various research on 'electromagnetic metamaterial absorbers'.

Electromagnetic metamaterials can possess unique electromagnetic attributes that can be explained with unusual values of effective material parameters such as magnetic permeability and electric permittivity. Smith, Padilla, and others have proposed that a broad range of electromagnetic responses can be achieved by arranging conductive scatterers ${ }^{5,6}$. Based on their research, many studies have been actively conducted for unnatural properties such as negative refractive index ${ }^{7,8}$, super resolution ${ }^{9,10}$, and wave absorption ${ }^{11-13}$. Especially, when applied to absorbers, electromagnetic metamaterials can improve the device performance and impart desirable qualities such as polarization-insensitivity, wide incident angle characteristics, multi-resonances, and multi-band absorption ${ }^{14-16}$. Due to the vastly increased freedom of material choice, electromagnetic metamaterial-based absorbers can have additional advantages in strength, elasticity, heat conduction, and other mechanical or chemical properties. However, there are still hurdles remaining in implementing these ideas in real devices and finding fabrication-friendly designs as well as practical and cost-effective fabrication methods is among the missing

\footnotetext{
${ }^{1}$ Department of Nano Mechatronics Engineering, University of Science and Technology (UST), Daejeon 34113, Republic of Korea. ${ }^{2}$ Department of Nano Manufacturing Technology, Korea Institute of Machinery and Materials (KIMM), Daejeon 34103, Republic of Korea. ${ }^{3}$ Advanced Cutting Tools Research Team, Daegu Mechatronics and Materials Institute (DMI), Daegu 42714, Republic of Korea. "4Department of Materials Science and Engineering, Korea Advanced Institute of Science and Technology (KAIST), Daejeon 34141, Republic of Korea. ${ }^{5}$ School of Materials Science and Engineering, University of Ulsan, Ulsan 44610, Republic of Korea. ${ }^{6}$ These authors contributed equally: Jun Sae Han and Tae-Jin Je. ${ }^{\varpi}$ email: jshan@kimm.re.kr; jtj@kimm.re.kr
} 
pieces. For example, the lumped resistor inclusions in some of the proposed metamaterial absorber designs are not ideal in terms of fabrication cost and durability. To solve these problems, many studied metamaterials using a polymeric conductive material including carbon or graphite with periodic patterns as a circuit and specific sheet resistance instead of using materials having lumped resistances ${ }^{14,17-20}$.

Fabrication of microwave metamaterials composed of conductive materials usually involve a screen-printing process or an inkjet printing process ${ }^{20-25}$. These processes can fabricate various shapes of circuit patterns (square loop $^{26-28}$, cross dipole ${ }^{29-31}$, fivefold ${ }^{23,25,32}$, square patch $^{33-35}$, and so on) on a dielectric substrate. Although printing processes are promising candidates for low-cost fabrication of microwave metamaterials, it is difficult to adjust the sheet resistance value for different parts of a pattern in a finely-controlled fashion. In addition, typical printing processes result in embossed (rather than engraved) patterns because they are additive process depositing new material on flat substrates. The embossed conductive materials are more prone to mechanical deformation or damage in the removing and curing process of the ink ${ }^{36}$. By contrast, engraved ink patterns on mechanical machined substrates allow a fine control of the thicknesses of different parts of the pattern on the same layer simply by controlling the machining depth. Moreover, rheology properties of inks have much less effects on final shapes of patterns because inks only fill engraved patterns.

The aim of this study was to develop and characterize the manufacturing process for a microwave absorber with resistive double square loop arrays. The developed process consisted of micro-end-milling, inking, and planing steps. End-milling means engraving double square loop arrays. Inking is filling conductive carbon ink to the engraved array structures. Planing is removing overfilled conductive ink and machining the planar surface. Under the established process, the fabricated microwave absorber having a thickness of $4.16 \mathrm{~mm}$ showed RCS reduction with reflectance less than $-10 \mathrm{~dB}$ in the range of $8.0-14.6 \mathrm{GHz}$.

\section{Experiments}

Design of the microwave absorber. A typical metamaterial absorber is composed of a metal-backed dielectric layer and a top resistive layer. The impedance of the metal-backed substrate can be calculated with the following equation:

$$
\eta_{\text {subs }}=i \frac{\eta_{0}}{n} \tan \left(\mathrm{nk}_{0} \mathrm{~d}\right)
$$

where $\mathrm{n}$ is the refractive index, $\eta_{0}$ is the impedance of free space (air), $\mathrm{d}$ is the thickness of the non-magnetic dielectric, and $k_{0}$ is the wave number in free space. With the concept of equivalent circuit model, the total impedance value of the whole absorber structure can be calculated with the following equation:

$$
\frac{1}{\eta}=\frac{1}{\eta_{s u b s}}+\frac{1}{\eta_{r}}
$$

where $\eta$ is the impedance of the whole absorber structure and $\eta_{r}$ is the impedance of the top resistive layer. The required impedance value of the top resistive layer can be calculated for the specific frequency range with the predetermined value of thickness and the dielectric index. The strategy to attain the required impedance value is very wide. Various designs including cross, ring, and patch for the resistive layer have been reported. We selected double square loop arrays and designed to have different sheet resistance for each square loop in order to confirm the performance of the developed manufacturing process due to high degrees of freedom in its shape, including length, width, and depth of each loop.

Figure la,b show a designed unit cell of microwave absorber. This absorber structure is optimized with a PSO (particle swarm optimization) algorithm applied to a Lumerical FDTD solver. The target performance of the absorber was set to be lower than $20 \%$ reflectance at 5-14 GHz, a sufficient performance to confirm the effectiveness of the manufacturing process developed in this study. Eight parameters were optimized during the process, including period, thickness of the structure, length, width, depth of each ring with a predetermined refractive index of substrate $n=1.732+0.00866 i$ and an ink conductivity $\sigma=2352.9 \mathrm{~S} / \mathrm{m}$.

The microwave absorber was designed to have three layers with resistive patterns, insulator material, and a metal sheet: (i) conductive carbon ink based on frequency selective surface layer, (ii) PMMA based insulating layer for the spacer to adjust the resonance position between incident microwave from radar and reflected microwave from ground, and (iii) metal sheet layer for reflection of transmitted microwave. This absorber material had a 144-number of double square loops in $150 \mathrm{~mm}^{2}$ area of PMMA material with a thickness of $4.16 \mathrm{~mm}$ as shown in Fig. 1c.

Figures 2 and 3 show the real part of the $\mathrm{E}_{\mathrm{x}}$ field and $\mathrm{Hz}$ field distributions at the middle plane of the double square loop. In the simulation settings, a plane wave was normally incident on the absorber structure and the electric field was on the $\mathrm{X}$ direction. Figure 2 shows electric and magnetic field distributions for $5.7 \mathrm{GHz}$ wave incidence. Figure 3 shows electric and magnetic field distributions for $12.7 \mathrm{GHz}$ where the reflectance dips occur. As shown in each figure, two sides of the ring parallel to the external electric field showed inductive behaviors while others showed capacitive behaviors. Due to these resonant behaviors of conductive rings at different frequencies, high absorption can occur. Simulation results for reflectance of the absorber structure are shown in Fig. 4. The designed structure showed an absorption performance more than $80 \%$ at $4.7-14.3 \mathrm{GHz}$.

Manufacturing process of the microwave absorber. Figure 5 shows the developed manufacturing process for the designed microwave absorber with resistive double square loop arrays on an insulator material. The developed process had four steps. The first step was a micro-end-milling process for engraving array structure on the surface of the insulator layer. The second step was an inking process for fill up the engraved array 
(a)

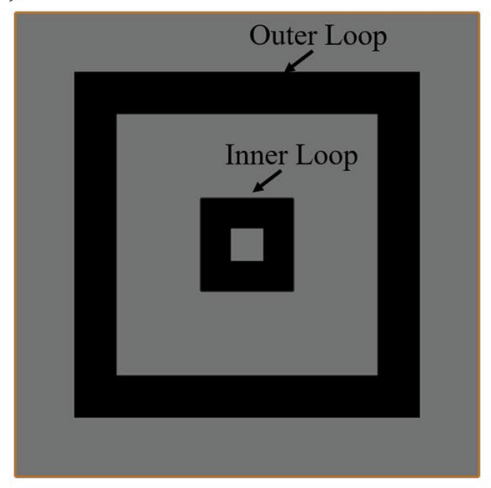

(b)

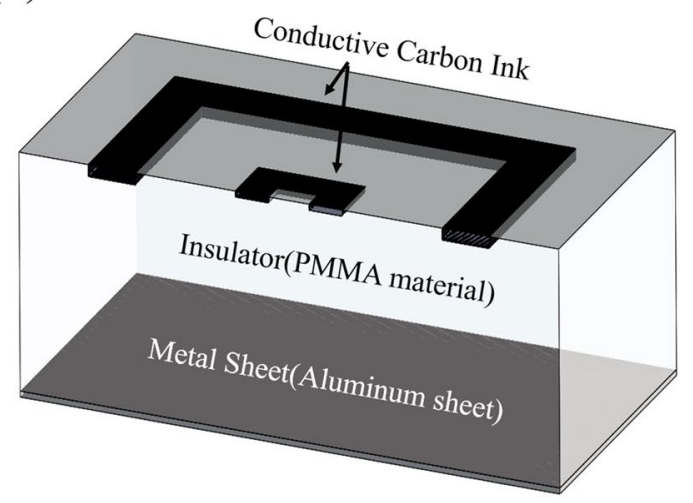

(c)

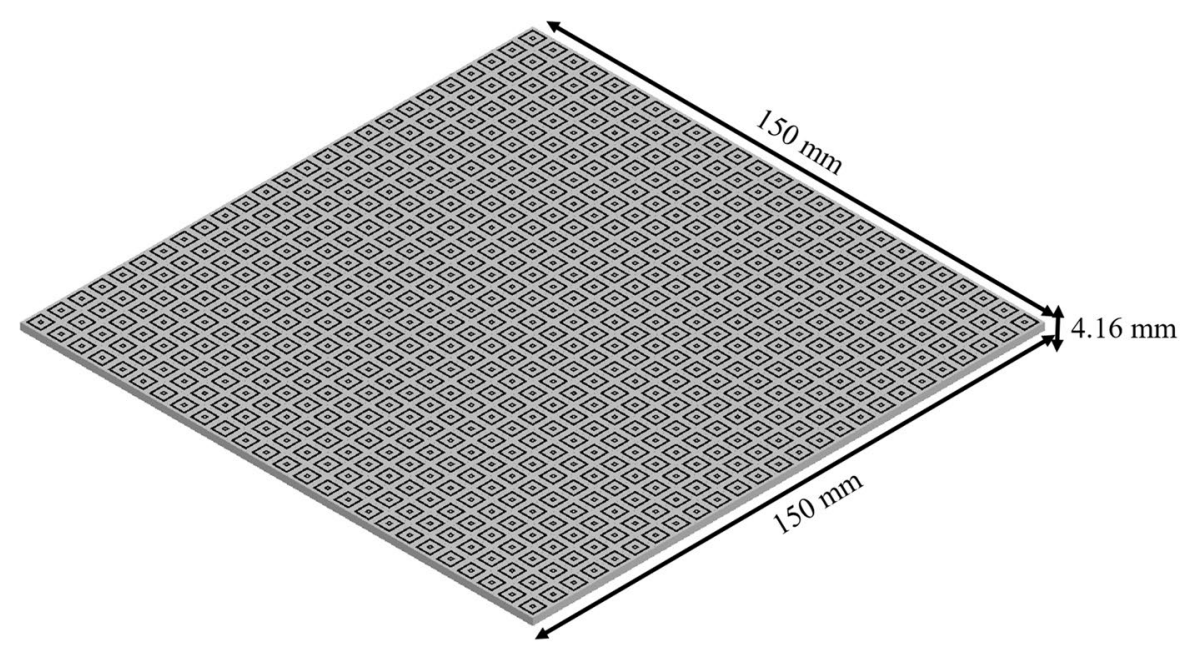

Figure 1. Structural design of the microwave absorber with resistive double square loop arrays. (a) Top view of unit-cell, (b) cross-section of the unit-cell, (c) full structure of the microwave absorber.
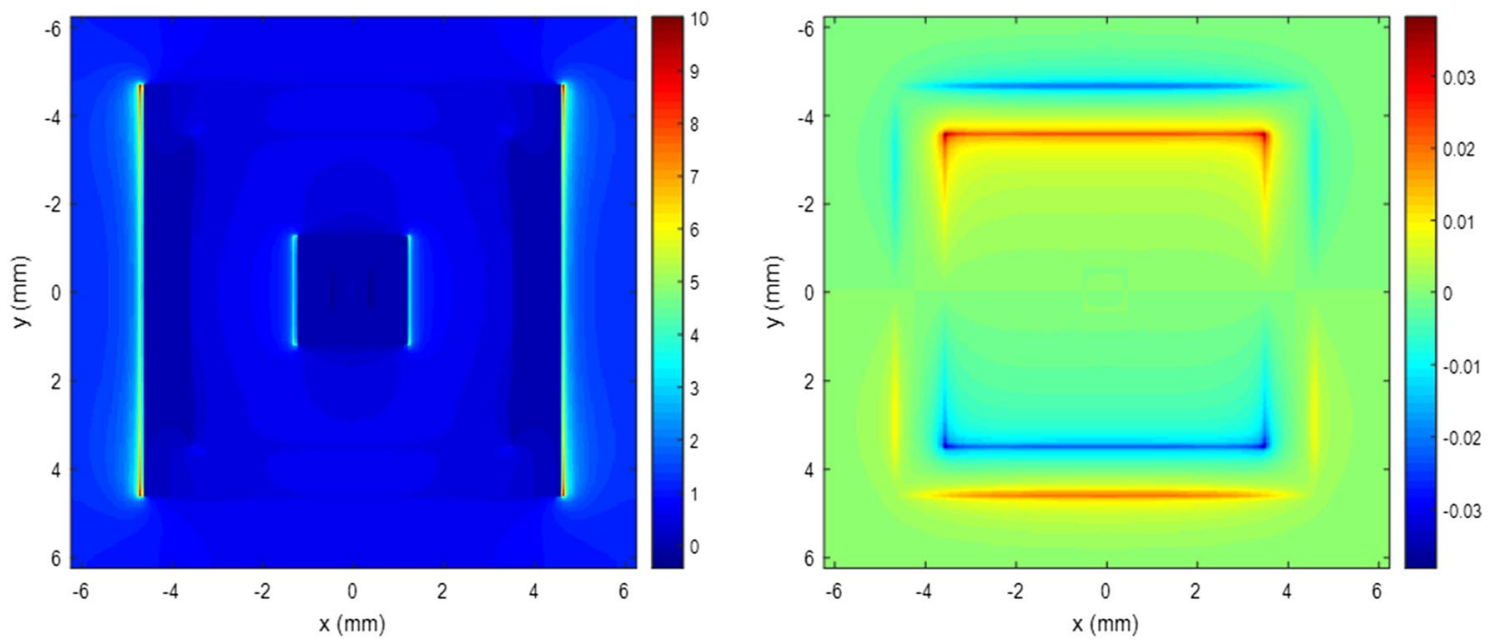

Figure 2. Electric and magnetic field distributions of a double square loop pattern at $5.7 \mathrm{GHz}$.

structure with the conductive ink. The third step was a planing process to remove the conductive ink overfilled in excess of the array structure. The final step was attaching a metal sheet to the bottom of the insulator layer. In this study, the PMMA material was used as an insulator layer. This is because it has advantages such as good machinability, appropriate refractive index, and superior transparency. The refractive index of PMMA was confirmed by simulation of magnetic and electric filed distributions. Transparency of PMMA has advantages in 

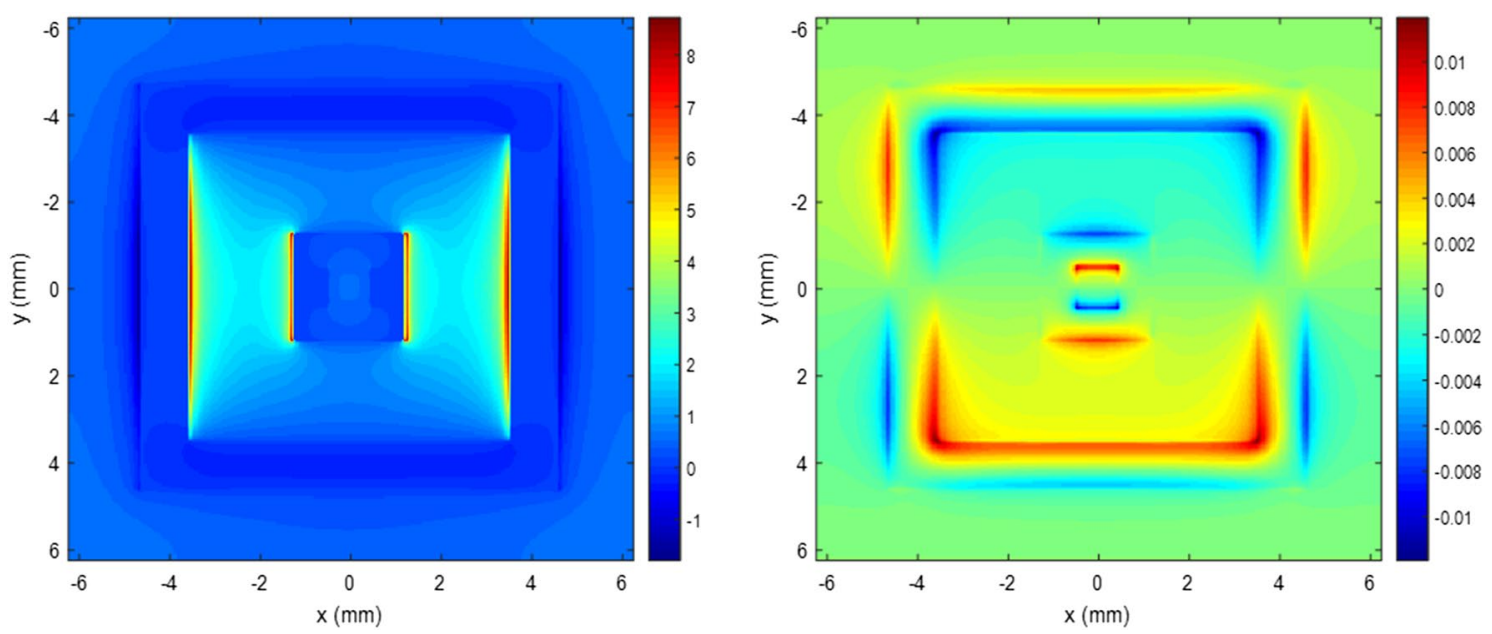

Figure 3. Electric and magnetic field distributions of a double square loop pattern at $12.7 \mathrm{GHz}$.

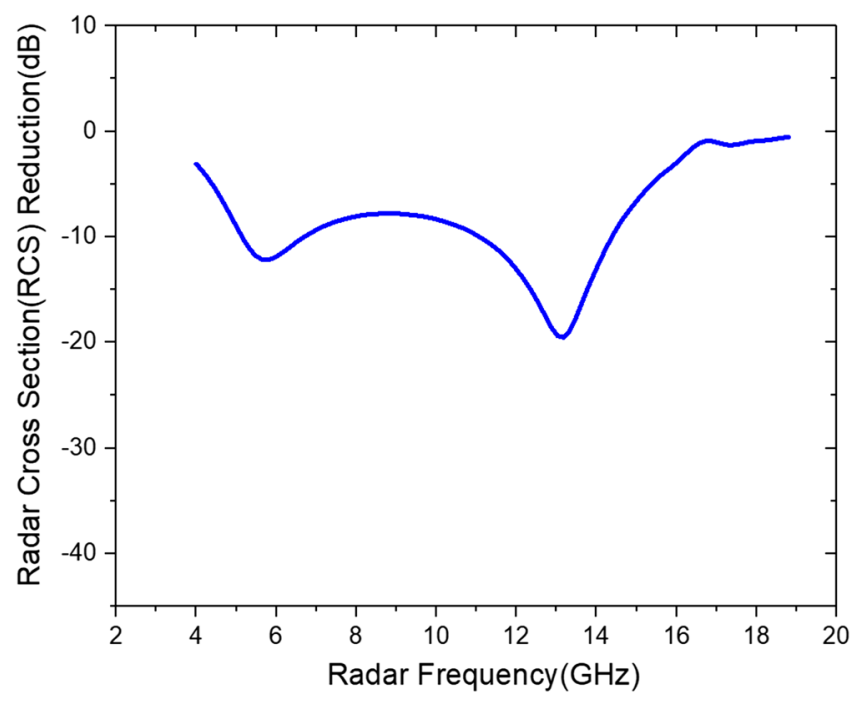

Figure 4. Simulated RCS reduction of the designed microwave absorber at 4 to $18 \mathrm{GHz}$.

confirming the residual conductive carbon ink on the microwave absorber after removing overfilled conductive ink by a planing process. For these reasons, the PMMA was chosen as the insulator material in this study. In the entire step, each processing condition was experimentally developed and optimized.

\section{Results and discussion}

Micro-end-milling process. As the first step, a flat PMMA workpiece was mechanically machined using an ultra-precision micro-end-milling system. Figure 6 shows an ultra-precision machining system having a threeaxis with $10 \mathrm{~nm}$ positioning resolution and air bearing spindle having a max rotation speed of 100,000 rpm. TiAlN (Titanium Aluminum Nitride) coated solid carbide with two flutes and a square end shape having a diameter of $400 \mu \mathrm{m}$ was used as a cutting tool for strong wear resistance and lubrication ability. Cutting oil of ISOPAR-H was spread to the endmill using a mist nozzle to prevent built-up edge caused by the characteristic of PMMA that could easily stick to the cutting tool surface because of cutting heat.

Optimized machining conditions are summarized in Table 1 . Since the machining quality by the end-milling process can greatly affect the performance of microwave absorption, quality optimization of roughness, shape, and size accuracy is needed by optimizing machining conditions. Especially, burr has a significantly degrading quality of roughness and shape accuracy of the engraved pattern. Burr can be easily formed on the edge and machined surface in the first and finish cuts. Moreover, the volume of burr tends to increase as more materials are removed at one time ${ }^{37}$. For these reasons, in order to minimize burr formation, the depth of cut was set to be $5 \mu \mathrm{m}$ for the first and finish cuts. The rough cut with a relatively low impact on the machining quality was applied to the remaining depth. Total cutting depths of each pattern were designed at $22 \mu \mathrm{m}$ for the inner pattern and $30 \mu \mathrm{m}$ for the outer pattern that included $5 \mu \mathrm{m}$ of the planing margin. Theoretical surface roughness after the end-milling process can be calculated with Eq. $(3)^{38}$. 


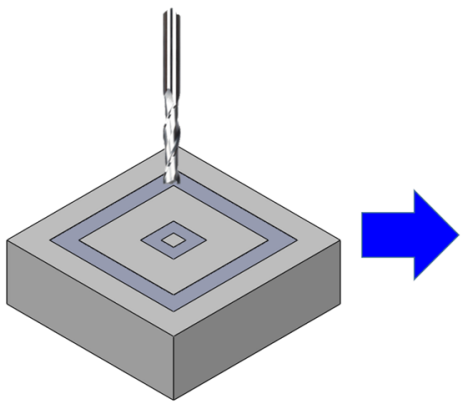

(I) Micro-end-milling process

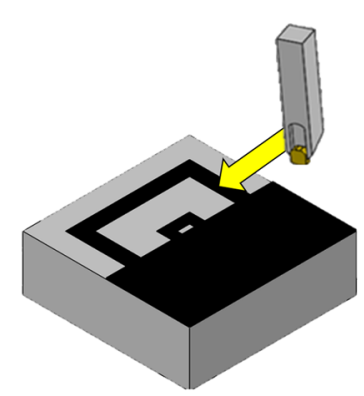

(III) Planing process
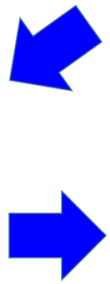

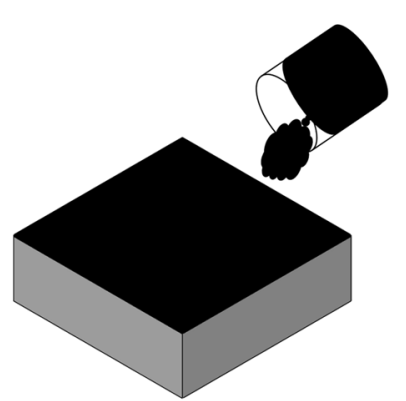

(II) Inking process
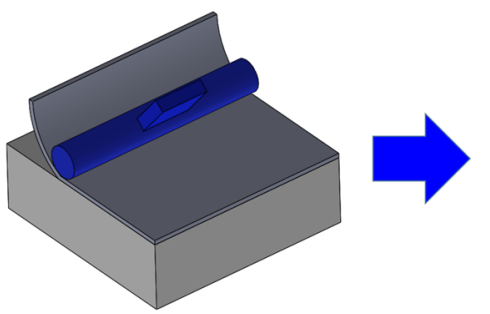

(IV) Attatching metal sheet

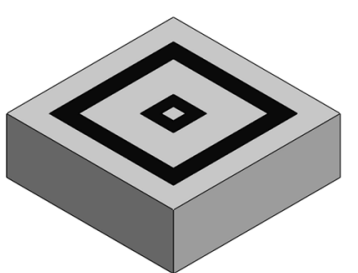

Microwave absorber

Figure 5. A schematic diagram showing the developed manufacturing processes for microwave absorber with resistive double square loop on a PMMA.

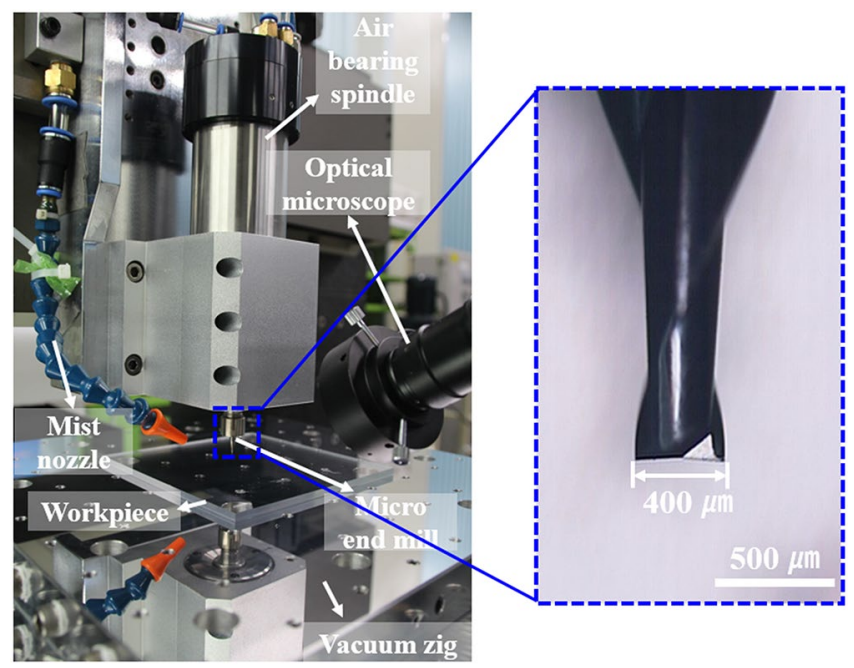

Figure 6. The micro-end-milling system for engraving of double square loop arrays on the PMMA workpiece and the cutting tool of TiAlN coated solid carbide.

\begin{tabular}{|l|l|l|l|l|l|}
\hline $\begin{array}{l}\text { Cutting tool rotation } \\
\text { speed (rpm) }\end{array}$ & \multicolumn{2}{|l|}{80,000} \\
\hline $\begin{array}{l}\text { Feed per revolution } \\
(\mu \mathrm{m} / \mathrm{rev})\end{array}$ & 9 & First cut & Rough cut & Finish cut & Total \\
\hline \multirow{4}{*}{ Cutting depth $(\mu \mathrm{m})$} & Inner pattern & 5 & 12 & 5 & 22 \\
\cline { 2 - 6 } & Outer pattern & 5 & 20 & 5 & 30 \\
\cline { 2 - 6 } & & & & \\
\hline
\end{tabular}

Table 1. Optimized machining conditions of the micro-end-milling process for double square array patterns on the PMMA surface. 

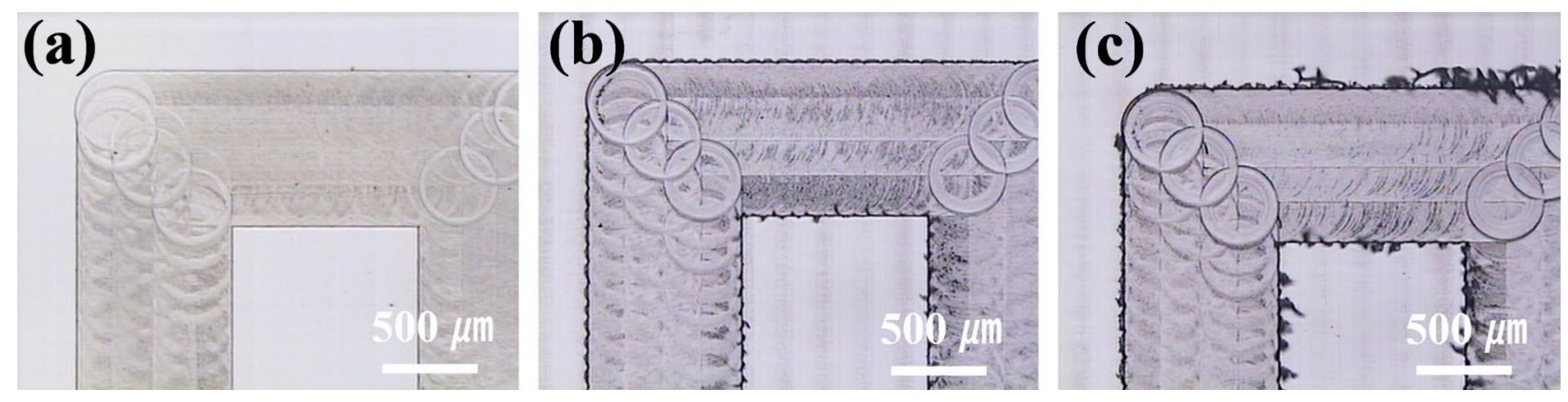

Figure 7. Microscope images of the machined inner pattern: (a) the first inner pattern, (b) the 72nd inner pattern, and (c) the 144th inner pattern.
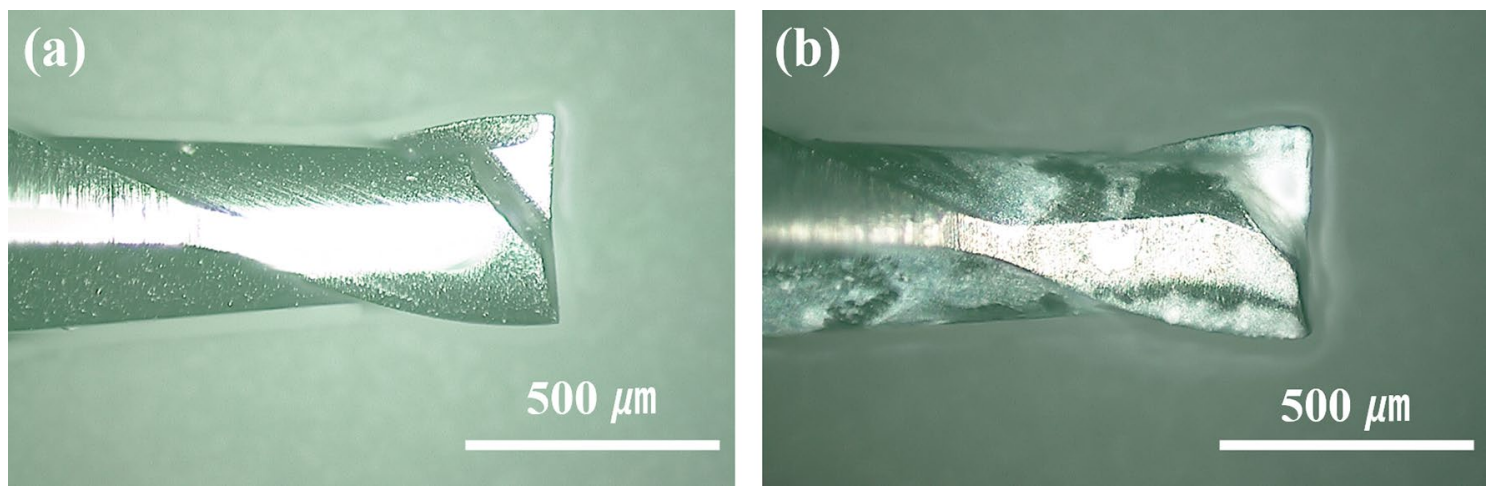

Figure 8. Microscope images of cutting tools before and after machining: (a) before machining and (b) after machining of the 144th double square loop.

$$
R_{a, e}=\frac{f_{t}^{2}}{32 \times\left(D / 2 \pm f_{t} \times n_{t} / \pi\right)}
$$

where $R_{a, e}$ is the average surface roughness machined by the micro-end-milling process, $f_{t}$ is the feed per revolution, $n_{t}$ is the number of teeth on the end-mill, and $D$ is edge diameter of the tool. The plus sign means an upmilling process and the minus sign means a down-milling process. The up-milling process was applied to obtain excellent surface roughness as minimizing burr formation. The rotation speed of the cutting tool and feed per revolution were applied to $80,000 \mathrm{rpm}$ and $9 \mu \mathrm{m} / \mathrm{rev}$, respectively. The theoretical surface roughness under the above conditions was calculated to be about $12.3 \mathrm{~nm}$.

Figure 7a,b,c show the first, 72nd, and 144th inner patterns of double square loops after the end-milling process, respectively. The first inner pattern showed clear edges with a smoothly machined surface. However, with increasing machining number of patterns, the size of the burr was also increased and the machined surface was roughened with a significant tool mark.

Figure 8a,b show cutting tools before and after machining of the 144th double square loop. Machinability of the cutting tool was remarkably decreased because the machined materials were adhered to the cutting edge. An ultrasonic cleaning method was additionally applied for removing the built-up edge on the cutting tool. The cleaning time was set to be 3 min after machining each of 24 patterns.

Figure 9 shows machined PMMA workpiece with 144-member double square patterns $(150 \mathrm{~mm} \times 150 \mathrm{~mm}$ in width and $4.05 \mathrm{~mm}$ in thickness). Widths of the machined inner and outer patterns were about $772.18 \mu \mathrm{m}$ and $1081.05 \mu \mathrm{m}$, respectively. Heights of the machined inner and outer patterns were about $22.05 \mu \mathrm{m}$ and $30.35 \mu \mathrm{m}$, respectively. Their average surface roughness was about $55 \mathrm{~nm}$. The surface roughness was larger than twice of theoretical value because factors such as vibration and machining environment were excluded from the theoretical formula. In consideration of these factors, we fabricated an excellent surface with an average roughness of less than $100 \mathrm{~nm}$. Also, machined patterns were successfully fabricated within $1 \%$ of machining error compared to the target size through an optimized end-milling process.

Inking and planing processes. As a second step, machined double square loop arrays on the surface of PMMA were filled with conductive carbon ink by the inking process. The most important factor in the inking process was the sheet resistance of conductive carbon ink. If the sheet resistance was different from the target value for generating electromagnetic field, the bandwidth of radar absorption could be changed or the performance of the RCS reduction could be degraded. The sheet resistance of the conductive carbon ink used in this experiment was controlled by mixing polymers of conventional carbon ink and silver paste with a solvent of thinner. Properties of the conductive carbon ink, the silver paste, and the developed conductive ink are pre- 

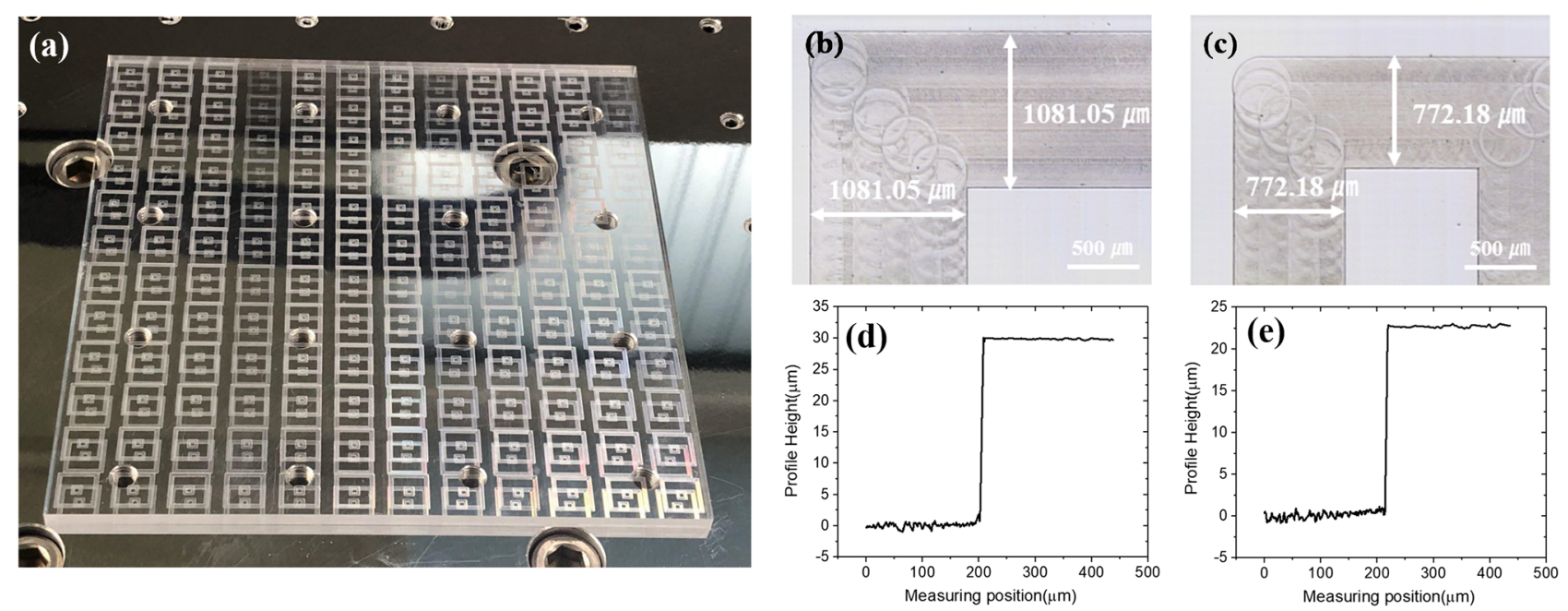

Figure 9. Measuring results of engraved double square loop on the PMMA workpiece by the micro-endmilling. (a) A camera image of machined PMMA workpiece after the end-milling process, (b) top view of the outer pattern, (c) top view of the inner pattern, (d) depth profile of the outer pattern, and (e) depth profile of the inner pattern.

\begin{tabular}{|l|l|l|l|l|l|}
\hline Type & Pigment & Solid ratio $(\%)$ & Viscosity $\left(\right.$ Poise $\left./ 25{ }^{\circ} \mathrm{C}\right)$ & $\begin{array}{l}\text { Sheet resistivity }(\boldsymbol{\Omega} / \mathbf{s q} \text { at } \\
\text { thickness of } 25 \boldsymbol{\mu m})\end{array}$ & $\begin{array}{l}\text { Manufacturer and model } \\
\text { name }\end{array}$ \\
\hline Carbon ink & Carbon, graphite & $40-43$ & $85-105$ & 50 & $\begin{array}{l}\text { SunChemical } \\
\text { GST4500 }\end{array}$ \\
\hline Silver paste & Silver & - & 230 & $4 \times 10^{-8}$ & $\begin{array}{l}\text { CANS } \\
\text { p-100 }\end{array}$ \\
\hline
\end{tabular}

Table 2. Properties of the conductive carbon ink and the silver paste.

sented in Table 2. The conductive carbon ink was GST 4500 manufactured by SunChemical Co. in the USA. It had a sheet resistance of $50 \Omega / \mathrm{sq}$ at $25 \mu \mathrm{m}$. The silver paste was p-100 manufactured by CANS Co. in JAPAN. It had a sheet resistance of $4 \times 10^{-8} \Omega / \mathrm{sq}$ at $25 \mu \mathrm{m}$. In order to achieve designed sheet resistance, conductive ink, silver paste, and thinner were mixed as a ratio of 47.5:1:1. The mixed conductive carbon ink had sheet resistance of $15 \Omega /$ sq at $25 \mu \mathrm{m}$.

For filling the ink, the machined PMMA workpiece was detached from the machining table of the micromilling system. The machined PMMA surface was then completely covered by manually pouring and spreading the developed conductive ink onto its surface. To completely cure the ink without deforming the workpiece by heating, the conductive ink was cured at room temperature $\left(23^{\circ} \mathrm{C}\right)$ for one day. The workpiece with the fully cured ink was remounted on the planing system that changed the cutting tool from micro-end-mill to single crystal diamond with a nose radius of $10 \mathrm{~mm}$.

In the third step, the over filled ink was removed using the planing process to completely isolate each pattern. In the planing process, the theoretical surface roughness was calculated with Eq. $(4)^{39}$.

$$
R_{a, p}=\frac{0.30321 \times f^{2}}{R / 2}
$$

where $R_{a, p}$ is the average surface roughness machined by the planing process, $f$ is the cutting pitch, and $R$ is the diameter of nose radius of the cutting tool. According to this equation, the roughness of the machined surface by the planing process was determined by the nose radius of the cutting tool and the cutting pitch. Since the nose radius of the cutting tool was a fixed variable, the cutting pitch was set to be $50 \mu \mathrm{m}$ to have a theoretical surface roughness of $31.25 \mathrm{~nm}$. Figure 10 shows the planing process to remove the overfilled ink on the PMMA workpiece. The overfilled ink was removed step by step as cutting depth was decreased from the highest position to the target thickness of $4 \mathrm{~mm}$.

The machined surface by the planing process was measured using a confocal laser microscope (Keyence VK-X1000). Figure 11a shows a 3D image of the PMMA surface after removing overfilled ink. Linear lines were formed on the PMMA surface with an equal direction to the cutting direction. Figure 11b shows roughness profile of this surface. $R_{a, p}$ is $38 \mathrm{~nm}$ which is close to the calculated value with Eq. (4). Figure 12a shows a 3D image of the carbon ink surface after removing the overfilled ink. Unlike the PMMA surface, the cured ink surface machined by the planing process was difficult to identify cutting lines. It was rougher than the PMMA surface. The $R_{a, p}$ of this surface was $68 \mathrm{~nm}$ as shown in Fig. 12b, which was close to twice of the value shown in Fig. 11b. This result was caused by the fact that the perfectly cured ink was more fragile than the PMMA. It was also caused by the irregular surface structure because of mixing various materials. Although the machined surface of PMMA and 

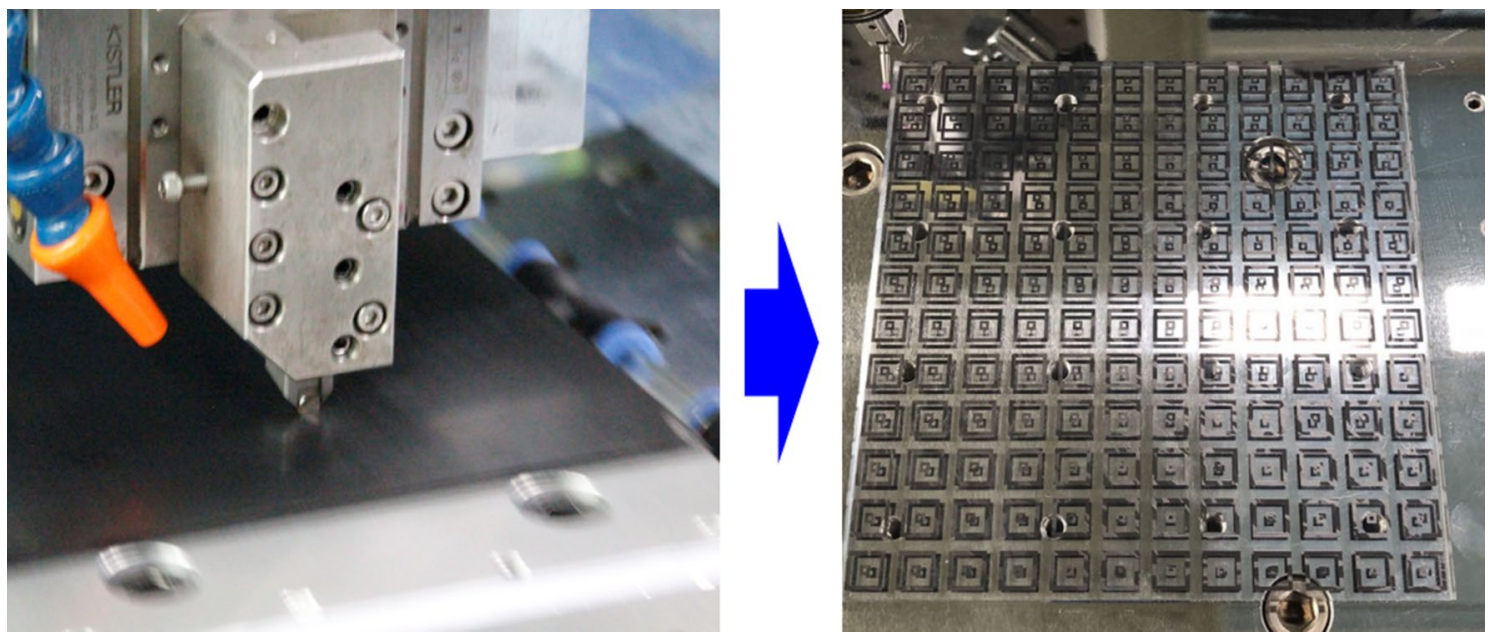

Figure 10. The planing process for removing the conductive carbon ink applied over the PMMA workpiece and the machined workpiece after removing the over filled ink.

(a)

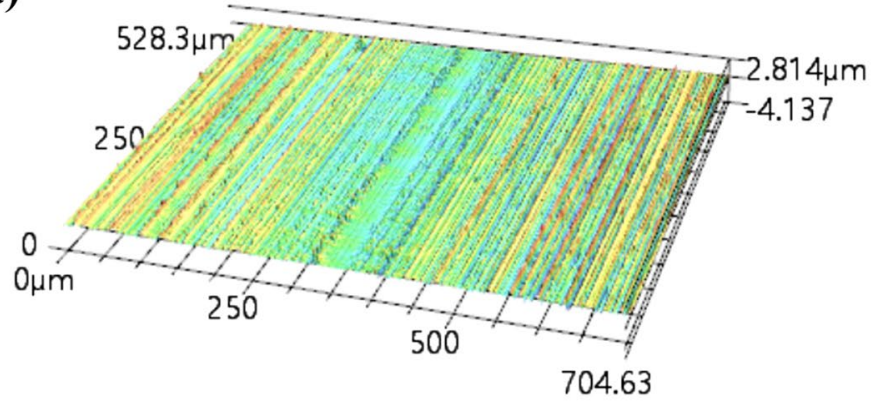

(b)

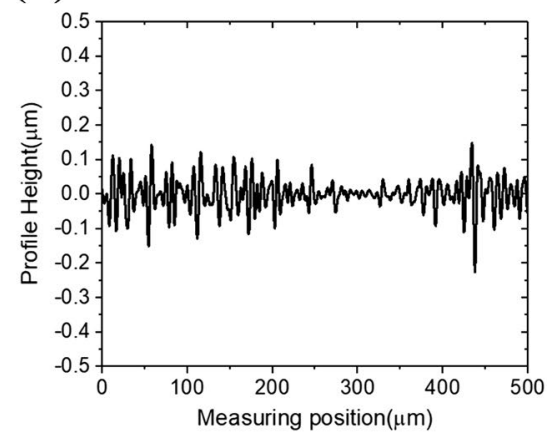

Figure 11. Confocal laser microscope measuring results of the PMMA surface after removing the overflowed conductive ink with the planing process. (a) 3D image of the PMMA surface after removing the overfilled conductive ink, (b) roughness profile of the PMMA surface after removing the overflowed conductive ink.

(a)

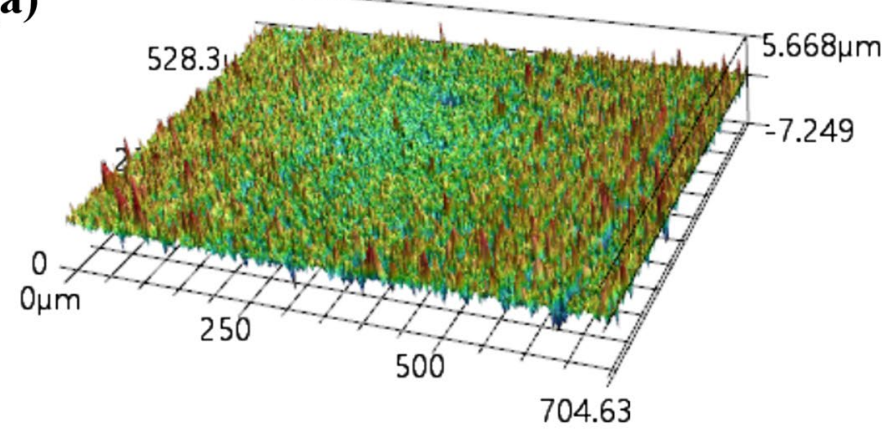

(b)

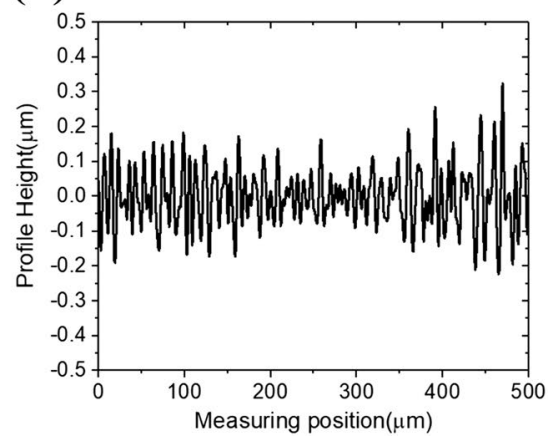

Figure 12. Confocal laser microscope measuring results of the resistive double square loops filled with the conductive ink machined by the planing process. (a) 3D image of ink surface after removing the overflowed conductive ink, (b) roughness profile of the ink surface after removing the overflowed conductive ink.

the surface of the conductive ink were different, both surfaces showed excellent characteristics with an average roughness of less than $100 \mathrm{~nm}$, which had less effect on the progress of microwaves. In addition, each array was completely filled and isolated without showing any residual ink on the surface. After the planing process as the final step, an absorption material for ultra-wide bandwidth microwave from C- to Ku-band (about 5-15 GHz) was fabricated by attaching a $160 \mu \mathrm{m}$ of the adhesive-applied aluminum sheet as a reflection layer on the back surface of PMMA workpiece for oscillating of the microwave. 
(a)
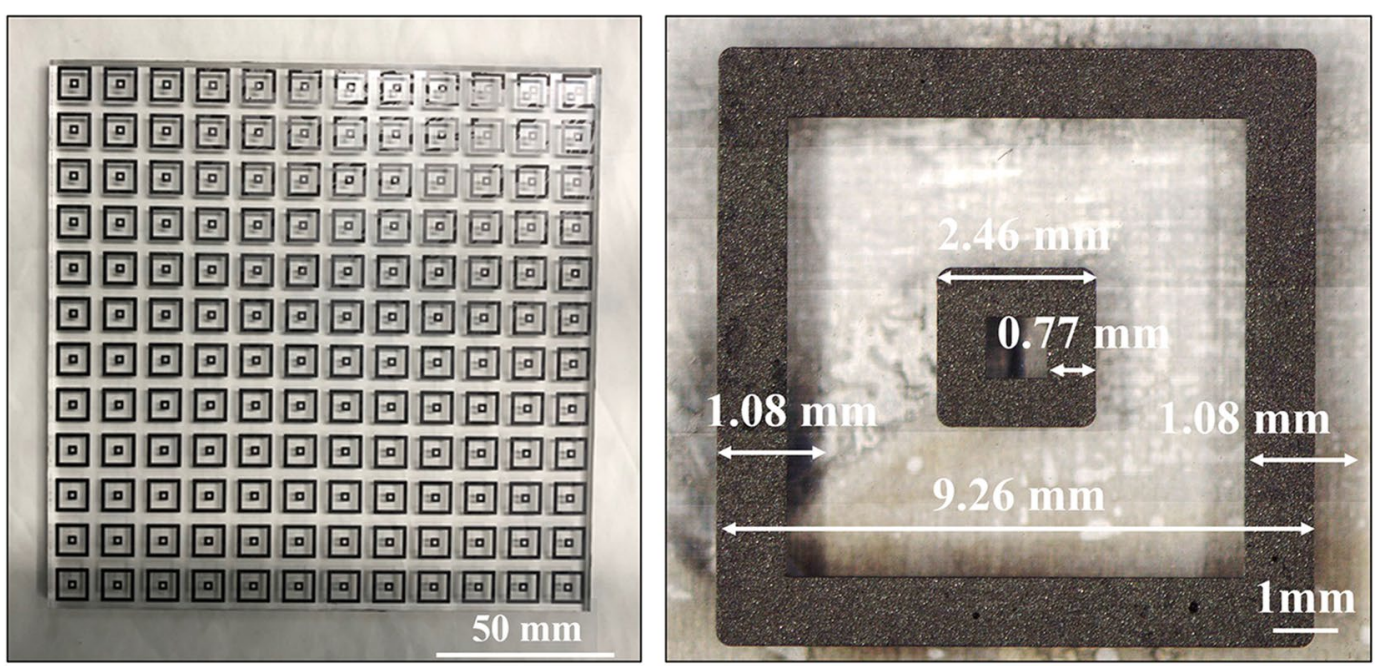

(b)

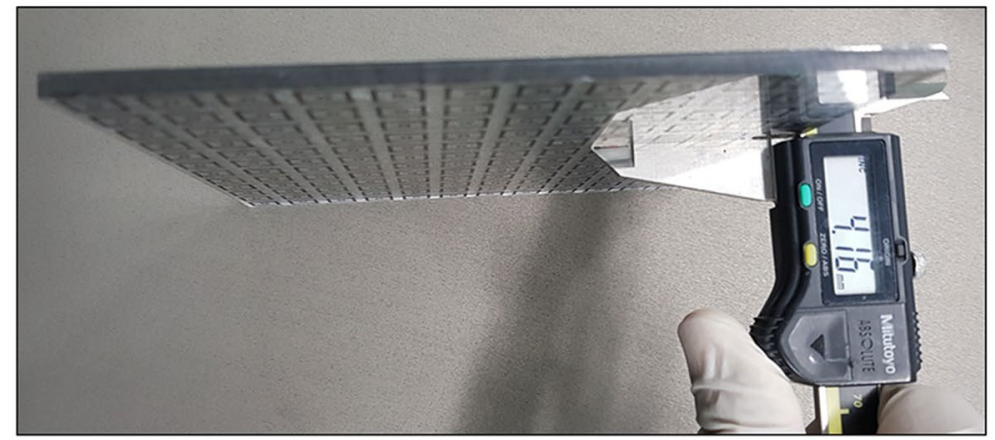

Figure 13. A microwave absorber fabricated by the developed manufacturing process. (a) Top view of the fabricated microwave absorber and enlarged image of resistive double square loop pattern with measuring the size and width of the pattern, (b) side view and measuring thickness of the microwave absorber.

Characterization of the microwave absorber with resistive double square loop arrays. Figure 13 shows fabricated microwave absorber with resistive double square loop arrays by the manufacturing process developed in this study. Figure 13a shows the fabricated microwave absorber with an area of $150 \mathrm{~mm}^{2}$ and an enlarged view of a double square pattern which has fully filled and cured conductive ink. Figure 13b shows the total thickness of $4.16 \mathrm{~mm}$ that comprises two polymer materials and a metal sheet. The PMMA material with a thickness of $4 \mathrm{~mm}$ conducts an isolation layer between the electromagnetic field and the ground. The resistive double square loops with array numbers of $12 \times 12$ generate an electromagnetic field with a distance of $4 \mathrm{~mm}$ from the ground surface. The size and the width of the inner pattern were about $2.46 \mathrm{~mm}$ and $0.77 \mathrm{~mm}$, respectively. The outer pattern size and width were about $9.26 \mathrm{~mm}$ and about $1.08 \mathrm{~mm}$, respectively. These patterns were machined within a size error of $1 \%$ compared to the target size and width. An aluminum sheet with a thickness of $0.16 \mathrm{~mm}$ reflected transmitted microwaves.

To characterize absorption properties, an RCS-measurement setup was constructed as shown in Fig. 14. A transmitting antenna with a focusing lens launched a Gaussian-like microwave beam to the target. A receiving antenna, also with a focusing lens, collected the reflected microwave from the target. These antennas were connected to a network analyzer. To evaluate the RCS reduction characteristics over a wide bandwidth, two sets of horn antennas, rated at $8.0-12.5 \mathrm{GHz}$ and $12.7-18.0 \mathrm{GHz}$, respectively, were used.

Figure 15 shows the measured reflectance as a function of the microwave frequency. The microwave absorbing performance of the fabricated microwave absorber was the highest near the center of the measured frequency range and gradually decreased towards the boundary. In the frequency band of $8.0-14.6 \mathrm{GHz}$, the measured reflectance was less than $-10 \mathrm{~dB}$. The minimum reflectance of $-41 \mathrm{~dB}$ was measured at $12.4 \mathrm{GHz}$. From simulations, it is expected that the frequency range with more than $10 \mathrm{~dB}$ attenuation may extend down to $\sim 5 \mathrm{GHz}$, but it requires further investigation. Although the measured RCS reduction performance showed a slight difference compared to simulated results, the tendency of absorption performance was similar. The cause of such difference between simulated and measured such as the maximum RCS reduction value and the resonance frequency was attributed to the non-uniformity of the applied conductive ink. This problem can be improved through a future study by optimizing the conductive ink application and curing process. 


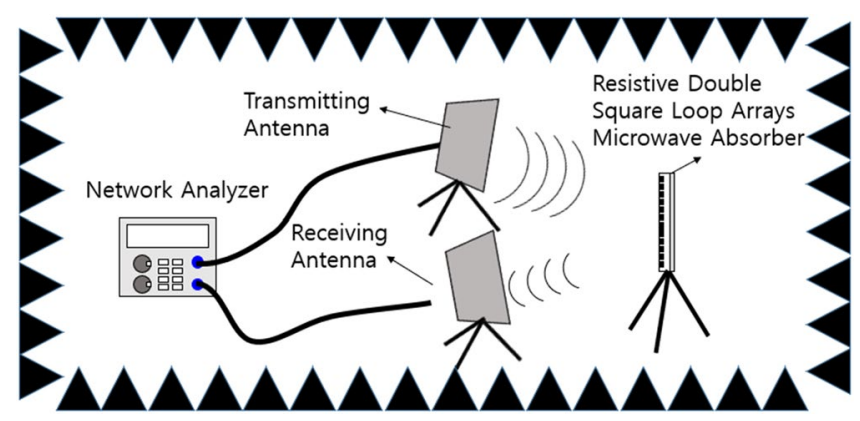

Figure 14. Schematic layout of the RCS reduction measurement system.

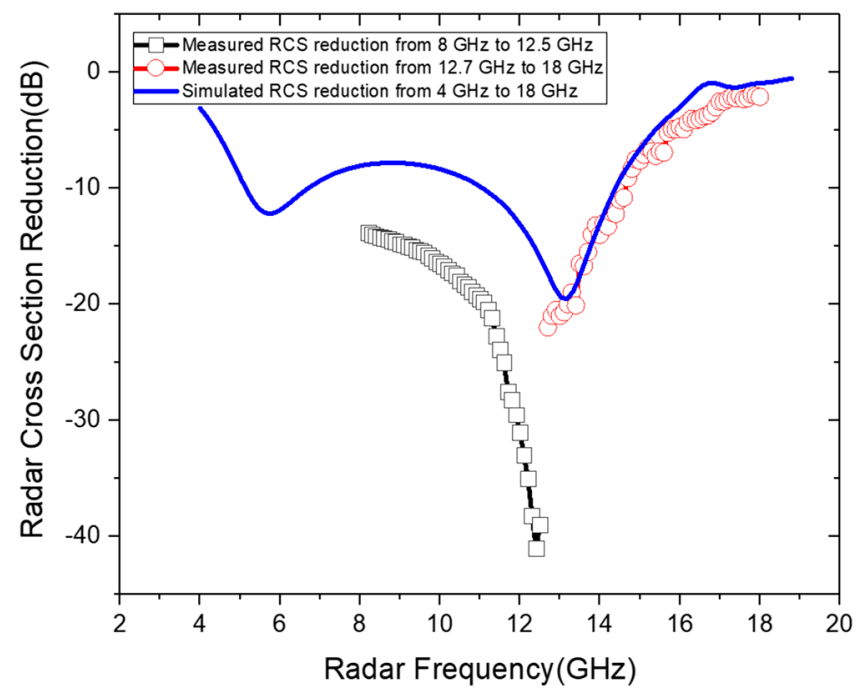

Figure 15. Measured and simulated RCS reduction of the fabricated microwave absorber according to radar frequency.

\begin{tabular}{|l|l|l|l|l|l|l|}
\hline Ref. & $\begin{array}{l}\text { Manufacturing process } \\
\text { (conductive material) }\end{array}$ & Pattern shape & Number of patterned layers & Thickness (mm) & Bandwidth (GHz) & $\begin{array}{l}\text { Maximum RCS reduction } \\
(\mathbf{d B})\end{array}$ \\
\hline 40 & Printing process (carbon ink) & Square loop array & 2 & 6.4 & $5-30.8$ & -42 \\
\hline 41 & Etching (copper) & Double square loop & 1 & 4.3 & $0.9-1.8$ & -41 \\
\hline 42 & (Carbon ink) & Square array & 2 & 3.5 & $8-18$ & -27.5 \\
\hline 43 & Silk printing (carbon ink) & Square array & 2 & 7.6 & $5.7-18$ & -49 \\
\hline 23 & Inkjet printing (CNT ink) & Jerusalem Cross + mesh grid & 2 & 5.1 & $8.2-44$ & -43 \\
\hline \multirow{2}{*}{ This work } & $\begin{array}{l}\text { Mechanical machining } \\
\text { process (carbon ink) }\end{array}$ & Double square loop & 1 & 4.2 & $8-14.6$ & -41 \\
\hline
\end{tabular}

Table 3. Comparison of layer number, pattern shape, thickness, bandwidth, and the maximum RCS reduction with other state-of-art broadband microwave absorbers.

Table 3 shows results of comparison in terms of layer number, pattern shape, thickness, bandwidth, and the maximum RCS reduction with other state-of-art broadband microwave absorbers. Previously reported microwave absorbers having multiple layers of resistive patterns have different sheet resistances or shapes on each layer to increase the absorbing performance and broaden the effective frequency range. On the other hand, the microwave absorbers by the proposed manufacturing process in this study can have patterns with different sheet resistances on a single layer. This advantage allows, thinner absorber designs with good absorption performance.

\section{Conclusion}

In this study, a manufacturing process was developed for a wide-bandwidth microwave absorber. The fabricated microwave absorber has 144 resistive double square patterns without connection among them. These patterns were precisely fabricated within a size error of $1 \%$ and a flat surface with an average roughness under $100 \mathrm{~nm}$. 
The developed microwave absorber showed a performance of RCS reduction with reflectance less than - $10 \mathrm{~dB}$ over 8 to $14.6 \mathrm{GHz}$ frequency range. To extend the RCS reduction performance to the entire Ku-band or higher frequencies and to make the performance more uniform across the frequency band, shapes of resistive array patterns and properties of the conductive carbon ink need to be optimized with further study.

Received: 23 February 2021; Accepted: 1 June 2021

Published online: 17 June 2021

\section{References}

1. Fan, M., He, Z. \& Pang, H. Microwave absorption enhancement of CIP/PANI composites. Synth. Met. 166, 1-6 (2013).

2. Meshram, M. R., Agrawal, N. K., Sinha, B. \& Misra, P. S. Transmission line modeling (TLM) for evaluation of absorption in ferrite based multi layer microwave absorber. In TENCON 2003. Conference on Convergent Technologies for Asia-Pacific Region, 1273246. https://doi.org/10.1109/tencon.2003.1273246 (2003).

3. Yan, S. J., Zhen, L., Xu, C. Y., Jiang, J. T. \& Shao, W. Z. Microwave absorption properties of $\mathrm{FeNi}_{3}$ submicrometre spheres and SiO $@$ $\mathrm{FeNi}_{3}$ core-shell structures. J. Phys. D Appl. Phys. 43, 245003 (2010).

4. Park, K.-Y. et al. Fabrication and electromagnetic characteristics of microwave absorbers containing carbon nanofibers and NiFe particles. Compos. Sci. Technol. 69, 1271-1278 (2009).

5. Smith, D. R. et al. Left-handed metamaterials. In Photonic Crystals and Light Localization in the 21st Century (ed. Soukoulis, C. M.) 351-371 (Springer, 2001). https://doi.org/10.1007/978-94-010-0738-2_25.

6. Padilla, W. J., Basov, D. N. \& Smith, D. R. Negative refractive index metamaterials. Mater. Today 9, 28-35 (2006).

7. Grbic, A. \& Eleftheriades, G. V. Experimental verification of backward-wave radiation from a negative refractive index metamaterial. J. Appl. Phys. 92, 5930-5935 (2002).

8. Ling, F., Zhong, Z., Huang, R. \& Zhang, B. A broadband tunable terahertz negative refractive index metamaterial. Sci. Rep. 8, 1-9 (2018).

9. Fang, N. \& Zhang, X. Imaging properties of a metamaterial superlens. In Proceedings of the 2nd IEEE Conference on Nanotechnology (IEEE, 0). https://doi.org/10.1109/nano.2002.1032233.

10. Rosenblatt, G. \& Orenstein, M. Perfect lensing by a single interface: defying loss and bandwidth limitations of metamaterials. Phys. Rev. Lett. 115, 195504 (2015).

11. Landy, N. I., Sajuyigbe, S., Mock, J. J., Smith, D. R. \& Padilla, W. J. Perfect metamaterial absorber. Phys. Rev. Lett. 100, 207402 (2008).

12. Luukkonen, O., Costa, F., Simovski, C. R., Monorchio, A. \& Tretyakov, S. A. A thin electromagnetic absorber for wide Incidence angles and both polarizations. IEEE Trans. Antennas Propag. 57, 3119-3125 (2009).

13. Colombi, A., Roux, P., Guenneau, S. \& Rupin, M. Directional cloaking of flexural waves in a plate with a locally resonant metamaterial. J. Acoust. Soc. Am. 137, 1783-1789 (2015).

14. Li, W. et al. Broadband composite radar absorbing structures with resistive frequency selective surface: optimal design, manufacturing and characterization. Compos. Sci. Technol. 145, 10-14 (2017).

15. Zhao, J. \& Cheng, Y. Ultrabroadband microwave metamaterial absorber based on electric SRR loaded with lumped resistors. J. Electron. Mater. 45, 5033-5039 (2016).

16. Bağmanc1, M. et al. Wideband metamaterial absorber based on CRRs with lumped elements for microwave energy harvesting. J. Microw. Power Electromagn. Energy. 52, 45-59 (2017).

17. Liu, T. et al. RCS Reduction of waveguide slot antenna with metamaterial absorber. IEEE Trans. Antennas Propag. 61, 1479-1484 (2013).

18. Wang, C. et al. Radar stealth and mechanical properties of a broadband radar absorbing structure. Compos. Part B Eng. 123, 19-27 (2017).

19. Liu, T. \& Kim, S.-S. Design of ultra wide-bandwidth double-layer electromagnetic wave absorbers with square-loop frequency selective surfaces. Microw. Opt. Technol. Lett. 60, 2013-2018 (2018).

20. Jaiswar, R. et al. A ultra-wideband thin microwave absorber using inkjet-printed frequency-selective surfaces combining carbon nanotubes and magnetic nanoparticles. Appl. Phys. A 125, 1-8 (2019).

21. Singh, G. et al. Fabrication of a non-wettable wearable textile-based metamaterial microwave absorber. J. Phys. D Appl. Phys. 52, 385304 (2019).

22. Bhati, A., Hiremath, K. R. \& Dixit, V. Bandwidth enhancement of Salisbury screen microwave absorber using wire metamaterial. Microw. Opt. Technol. Lett. 60, 891-897 (2018).

23. Jaiswar, R. et al. Inkjet-printed frequency-selective surfaces based on carbon nanotubes for ultra-wideband thin microwave absorbers. J. Mater. Sci. Mater. Electron. 31, 2190-2201 (2019).

24. Zabri, S. N., Cahill, R., Conway, G. \& Schuchinsky, A. Inkjet printing of resistively loaded FSS for microwave absorbers. Electron. Lett. 51, 999-1001 (2015).

25. Momeni-Nasab, M., Bidoki, S. M., Hadizadeh, M. \& Movahhedi, M. Ink-jet printed metamaterial microwave absorber using reactive inks. AEU Int. J. Electron. Commun. 123, 153259 (2020).

26. Liu, T. \& Kim, S.-S. Design of wide-bandwidth electromagnetic wave absorbers using the inductance and capacitance of a square loop-frequency selective surface calculated from an equivalent circuit model. Opt. Commun. 359, 372-377 (2016).

27. Tak, J. \& Choi, J. A wearable metamaterial microwave absorber. IEEE Antennas Wirel. Propag. Lett. 16, 784-787 (2017).

28. Hao, S. et al. Broadband microwave absorber by direct drawing metamaterial on paper. In 2019 International Conference on Microwave and Millimeter Wave Technology (ICMMT) (IEEE, 2019). https://doi.org/10.1109/icmmt45702.2019.8992265.

29. Kundu, D., Mohan, A. \& Chakrabarty, A. Single-layer wideband microwave absorber using array of crossed dipoles. IEEE Antennas Wirel. Propag. Lett. 15, 1589-1592 (2016).

30. Liu, H.-T., Cheng, H.-F., Chu, Z.-Y. \& Zhang, D.-Y. Absorbing properties of frequency selective surface absorbers with cross-shaped resistive patches. Mater. Des. 28, 2166-2171 (2007).

31. Sarkhel, A. \& Bhadra Chaudhuri, S. R. Compact quad-band polarization-insensitive ultrathin metamaterial absorber with wide angle stability. IEEE Antennas Wirel. Propag. Lett. 16, 3240-3244 (2017).

32. Yoo, M., Kim, H. K., Kim, S., Tentzeris, M. \& Lim, S. Silver nanoparticle-based inkjet-printed metamaterial absorber on flexible paper. IEEE Antennas Wirel. Propag. Lett. 14, 1718-1721 (2015).

33. Dewantari, A. \& Munir, A. Bandwidth enhancement of artificial magnetic conductor-based microwave absorber using square patch corner cutting. In 2012 th International Conference on Telecommunication Systems, Services, and Applications (TSSA) (IEEE, 2012). https://doi.org/10.1109/tssa.2012.6366053.

34. Li, Y., Li, W., Wang, Y., Cao, J. \& Guan, J. Refractory metamaterial microwave absorber with strong absorption insensitive to temperature. Adv. Opt. Mater. 6, 1800691 (2018).

35. Li, M., Muneer, B., Yi, Z. \& Zhu, Q. A broadband compatible multispectral metamaterial absorber for visible, near-infrared, and microwave bands. Adv. Opt. Mater. 6, 1701238 (2018). 
36. Hyun, W. J., Secor, E. B., Hersam, M. C., Frisbie, C. D. \& Francis, L. F. High-resolution patterning of graphene by screen printing with a silicon stencil for highly flexible printed electronics. Adv. Mater. 27, 109-115 (2014).

37. Korkmaz, E., Onler, R. \& Ozdoganlar, O. B. Micromilling of poly(methyl methacrylate, PMMA) using single-crystal diamond tools. Procedia Manuf. 10, 683-693 (2017).

38. Montgomery, D. \& Altintas, Y. Mechanism of cutting force and surface generation in dynamic milling. J. Manuf. Sci. Eng. Trans. ASME 113, 160-168 (1991).

39. Knight, W. A. \& Boothroyd, G. Fundamentals of Metal Machining and Machine Tools (CRC Press, 2019).

40. Huang, X. et al. Calculations of a wideband metamaterial absorber using equivalent medium theory. J. Phys. D Appl. Phys. 49, 325101 (2016).

41. Khalid, N. K. B. A. \& Seman, F. B. C. Double square loop frequency selective surface (FSS) for GSM shielding. In Lecture Notes in Electrical Engineering 223-229 (Springer International Publishing, 2014). https://doi.org/10.1007/978-3-319-07674-4_23.

42. Wang, C. et al. Radar stealth and mechanical properties of a broadband radar absorbing structure. Compos. B Eng. 123, 19-27 (2017).

43. Huang, Y. et al. Optimization of flexible multilayered metastructure fabricated by dielectric-magnetic nano lossy composites with broadband microwave absorption. Compos. Sci. Technol. 191, 108066 (2020).

\section{Acknowledgements}

This work was supported by the Center for Advanced Meta-Materials(CAMM) funded by the Ministry of Science and ICT as Global Frontier Project(CAMM- No. 2019M3A6B3031046). The work was also funded by the Technology Innovation Program of Ministry of Trade, Industry \& Energy(MOTIE, Korea) (20003945, Development of Core manufacturing technology of 4-axes ultra-precision machining system and lens module for next-generation mobile lens having about 100nm shape accuracy).

\section{Author contributions}

Conceptualization, T.J.Je., J.S.Han., E.c.Jeon., J.Y.Jeong.; Machining process, J.Y.Jeong., T.J.Je.; Inking process, J.S.Han., J.R.Lee.; Design of microwave absorber; H.J.Park., J.K.Jung., J.H.Shin.; Measuring machined pattern, J.Y.Jeong., J.R.Lee., D.S.Choi.; Measuring RCS reduction of microwave absorber, E.c.Jeon., H.J.Park., J.K.Jung., J.H.Shin.; Writing-original draft preparation, J.Y.Jeong., H.J.Park.; Writing-review and editing, J.Y.Jeong., J.S.Han., T.J.Je., J.H.Shin.; Supervision, J.S.Han., T.J.Je.; Project administration, D.S.Choi.; Funding acquisition, D.S.Choi. All authors have read and agreed to the published version of the manuscript.

\section{Competing interests}

The authors declare no competing interests.

\section{Additional information}

Correspondence and requests for materials should be addressed to J.S.H. or T.-J.J.

Reprints and permissions information is available at www.nature.com/reprints.

Publisher's note Springer Nature remains neutral with regard to jurisdictional claims in published maps and institutional affiliations.

(c) (i) Open Access This article is licensed under a Creative Commons Attribution 4.0 International cc) License, which permits use, sharing, adaptation, distribution and reproduction in any medium or format, as long as you give appropriate credit to the original author(s) and the source, provide a link to the Creative Commons licence, and indicate if changes were made. The images or other third party material in this article are included in the article's Creative Commons licence, unless indicated otherwise in a credit line to the material. If material is not included in the article's Creative Commons licence and your intended use is not permitted by statutory regulation or exceeds the permitted use, you will need to obtain permission directly from the copyright holder. To view a copy of this licence, visit http://creativecommons.org/licenses/by/4.0/.

(C) The Author(s) 2021 